\title{
ISAR-REACT 5 - What have we learned?
}

\author{
Jacek Kubica ${ }^{1}$, Miłosz Jaguszewski ${ }^{2}$ \\ ${ }^{1}$ Department of Cardiology and Internal Medicine, Collegium Medicum, \\ Nicolaus Copernicus University, Bydgoszcz, Poland \\ ${ }^{2}$ First Department of Cardiology, Medical University of Gdansk, Poland
}

\begin{abstract}
Paris, France 2019 several days ago, the results of the ISAR-REACT 5 were finally presented during the European Society of Cardiology ESC Congress 2019 in Paris. The Intracoronary Stenting and Antithrombotic Regimen: Rapid Early Action for Coronary Treat-

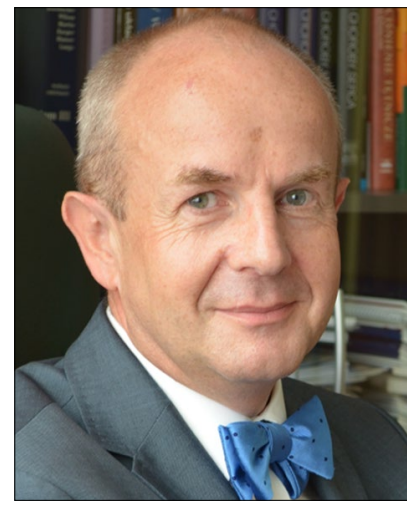
ment (ISAR-REACT) 5 trial was an investigator-initiated, phase 4, multicenter, randomized, open-label trial [1]. The study was designed to demonstrate the superiority of ticagrelor $v s$. prasugrel; in contrast, the main finding of this trial was the reported superiority of prasugrel over ticagrelor with respect to the composite endpoint of death, myocardial infarction, or stroke 1 year after randomization, in patients with acute coronary syndromes. Reduced incidence of the composite endpoint in the prasugrel arm was not associated with an increased risk of bleeding.

However, quite the opposite results were commonly expected based on the existing body of evidence from the previously published trials.

The ISAR-REACT 5 trial did not directly compare two antiplatelet agents, but rather different antiplatelet strategies mimicking the ESC guidelines, but not quite so [2-4]. Thus, the results do not reflect current clinical practice.

In our opinion, the study design and its organization lead to a bias that is difficult to estimate due to several reasons. Both the open-label design of the trial and the fact that it was performed only in two countries with a disproportion of enroll-

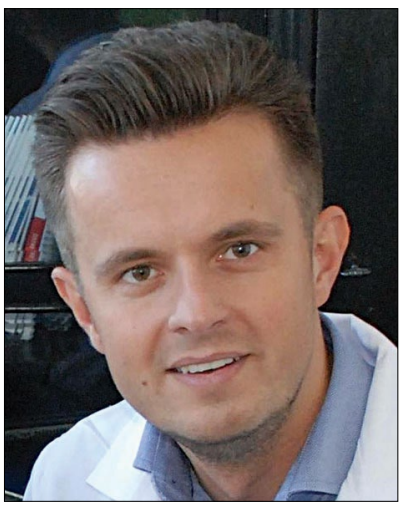

ing centers $(21$ centers in Germany and 2 centers in Italy) could have had some, however probably minor, impact on the outcomes. On the other hand, the adherence to the trial medication could have had much more influence on the results. The stated non-adherence to the treatment of $0.9 \%$ in prasugrel arm and $0.4 \%$ in ticagrelor arm seem to be unequivocally underestimated as patients were followed up mainly by telephone only (83\% of contacts), at hospital or by outpatient visit (10\%), or by using structured follow-up letter (7\%). Moreover, after the in-hospital phase of the trial commercially available ticagrelor or prasugrel were prescribed by the physician and had to be purchased by the patients themselves. No specific method for adherence evaluation, i.e. medication event monitoring systems, pill count, or drug availability according to the purchase of prescribed drugs etc., was reported. As the patientreported, drug intake was previously shown to be misleading, the true adherence to trial medication was probably much lower than that reported by the authors $[5,6]$.

The intention-to-treat analysis, i.e. with the inclusion of all patients according to the randomly assigned trial group, irrespective of the actual treatment received, is a widely accepted method for such kind of clinical studies. In this particular trial, however, such design might have led to serious distortion of the results, as only 1602 of 2012 and 1596 of 2006 subjects in ticagrelor and

Address for correspondence: Prof. Jacek Kubica, Department of Cardiology and Internal Medicine, Collegium Medicum, Nicolaus Copernicus University, ul. M. Skłodowskiej-Curie 9, 85-094 Bydgoszcz, Poland, tel: +48 52 5854023, fax: +4852 5854024, e-mail: jkubica@cm.umk.pl 
prasugrel groups, respectively, were discharged from the hospital on the allocated medication. Moreover, additional 243 and 199 patients in each arm, respectively, discontinued study medication after discharge, and finally, 19 and 18 patients were lost to follow-up in each group. The intention-to-treat principle applied in the ISAR-REACT 5 has led to the inclusion of at least 1262 patients in final analysis, who were not treated with the assigned medication (653 of 2012 subjects $(32.5 \%)$ and 609 of 2006 subjects $(30.4 \%)$ in ticagrelor and prasugrel arms, respectively).

A primary endpoint (the composite endpoint of all-cause death, myocardial infarction, or stroke) at 1 year after randomization, occurred in 184 of 2012 patients $(9.3 \%)$ in the ticagrelor group and in 137 of 2006 patients (6.9\%) in the prasugrel group (HR, 1.36 ; $95 \%$ CI, 1.09 to $1.70 ; \mathrm{P}=0.006$ ). Taking into account that the analysis of 4018 patients included 1262 (31.4\%) who were supposed to be on study medication, whereas they were not treated according to the study protocol, the absolute difference in primary endpoint incidence of 47 events can hardly be considered relevant.

In the analysis of the primary endpoint occurrence among subjects discharged on the study medication (1602 patients on ticagrelor and 1596 patients on prasugrel) in the period from discharge to the time of discontinuation of treatment or end of follow-up ("on treatment" analysis), the difference between the arms has been shown not to be significant (92 events in the ticagrelor group and 71 in the prasugrel group; HR, 1.34; 95\% CI, 0.98-1.82).

The observed bleeding rates are also the subject of questions. In the modified intention-totreat analysis the safety endpoint (major bleeding BARC type 3 through 5) was observed in 95 patients $(5.4 \%)$ in ticagrelor arm and in 80 patients $(4.8 \%)$ in the prasugrel arm (HR, 1.12; 95\% CI, 0.83 to $1.51 ; \mathrm{P}=0.46$ ). The absolute difference of 15 events between the groups, while as many as 233 of 2006 patients (11.6\%) from prasugrel group and only 23 of 2012 patients (1.1\%) from the ticagrelor group were excluded from this analysis without any justification, is difficult to interpret.
Thus, the result of both efficacy and safety needs to be carefully validated.

In conclusion, the ISAR-REACT 5 trial, indeed instead of providing answers, rather rises questions. Trying to answer the title question: "ISAR-REACT 5 - What have we learned?" we found ourselves in trouble, as we can not provide any definitive statement.

\section{Conflict of interest: Lecture honoraria}

\section{References}

1. Schüpke S, Neumann FJ, Menichelli M, et al. ISAR-REACT 5 Trial Investigators. Ticagrelor or Prasugrel in Patients with Acute Coronary Syndromes. N Engl J Med. 2019 [Epub ahead of print], doi: 10.1056/NEJMoa1908973, indexed in Pubmed: 31475799 .

2. Ibanez B, James S, Agewall S, et al. ESC Scientific Document Group. 2017 ESC Guidelines for the management of acute myocardial infarction in patients presenting with ST-segment elevation: The Task Force for the management of acute myocardial infarction in patients presenting with ST-segment elevation of the European Society of Cardiology (ESC). Eur Heart J. 2018; 39(2): 119-177, doi: 10.1093/eurheartj/ehx393, indexed in Pubmed: 28886621.

3. Roffi M, Patrono C, Collet JP, et al. ESC Scientific Document Group. 2015 ESC Guidelines for the management of acute coronary syndromes in patients presenting without persistent ST-segment elevation: Task Force for the Management of Acute Coronary Syndromes in Patients Presenting without Persistent ST-Segment Elevation of the European Society of Cardiology (ESC). Eur Heart J. 2016; 37(3): 267-315, doi: 10.1093/eurheartj/ /ehv320, indexed in Pubmed: 26320110.

4. Valgimigli M, Bueno H, Byrne RA, et al. ESC Scientific Document Group, ESC Committee for Practice Guidelines (CPG), ESC National Cardiac Societies . 2017 ESC focused update on dual antiplatelet therapy in coronary artery disease developed in collaboration with EACTS: The Task Force for dual antiplatelet therapy in coronary artery disease of the European Society of Cardiology (ESC) and of the European Association for CardioThoracic Surgery (EACTS). Eur Heart J. 2018; 39(3): 213-260, doi: 10.1093/eurheartj/ehx419, indexed in Pubmed: 28886622.

5. Shi L, Liu J, Fonseca V, et al. Correlation between adherence rates measured by MEMS and self-reported questionnaires: a meta-analysis. Health Qual Life Outcomes. 2010; 8: 99, doi: 10.1186/1477-7525-8-99, indexed in Pubmed: 20836888.

6. Kubica A, Kasprzak M, Obońska K, et al. Discrepancies in assessment of adherence to antiplatelet treatment after myocardial infarction. Pharmacology. 2015; 95(1-2): 50-58, doi: 10.1159/000371392, indexed in Pubmed: 25592409. 